\title{
Trends and results in treatment of gastric cancer over last two decades at single East European centre: a cohort study
}

\author{
Antanas Mickevicius ${ }^{1 *}$, Povilas Ignatavicius ${ }^{1,2}$, Rytis Markelis ${ }^{1}$, Audrius Parseliunas ${ }^{1}$, Dainora Butkute ${ }^{3}$,
} Mindaugas Kiudelis ${ }^{1}$, Zilvinas Endzinas ${ }^{1}$, Almantas Maleckas ${ }^{1}$ and Zilvinas Dambrauskas ${ }^{1,2}$

\begin{abstract}
Background: A steady decline in gastric cancer mortality rate over the last few decades is observed in Western Europe. However it is still not clear if this trend applies to Eastern Europe where high incidence rate of gastric cancer is observed.

Methods: This was a retrospective non-randomized, single center, cohort study. During the study period 557 consecutive patients diagnosed with gastric cancer in which curative operation was performed met the inclusion criteria. The study population was divided into two groups according to two equal time periods: 01-01-1994 - 31-12-2000 (Group I - 273 patients) and 01-01-2001 - 31-12-2007 (Group II - 284 patients). Primary (five-year survival rate) and secondary (postoperative complications, 30-day mortality rate and length of hospital stay) endpoints were evaluated and compared.

Results: Rate of postoperative complications was similar between the groups, except for Grade III (Clavien-Dindo grading system for the classification of surgical complications) complications that were observed at significantly lower rates in Group II (26 (9.5\%) vs. 11 (3.9\%), $p=0.02)$. Length of hospital stay was significantly $(p=0.001)$ shorter $(22.6 \pm 28.9$ vs. $16.2 \pm 17.01$ days) and 30-day mortality was significantly $(p=0.02)$ lower $(15(5.5 \%)$ vs. $4(1.4 \%))$ in Group II. Similar rates of gastric cancer related mortality were observed in both groups ( $92.3 \%$ vs. $90.7 \%)$. However survival analysis revealed significantly $(p=0.02)$ better overall 5-year survival rate in Group II (35.6\%, 101 of 284) than in Group I (23.4\%, 64 of 273). There was no difference in 5-year survival rate when comparing different TNM stages.

Conclusions: Gastric cancer treatment results remain poor despite decreasing early postoperative mortality rates, shortening hospital stay and improved overall survival over the time. Prognosis of treatment of gastric cancer depends mainly on the stage of the disease. Absence of screening programs and lack of clinical symptoms in early stages of gastric cancer lead to circumstances when most of the patients presenting with advanced stage of the disease can expect a median survival of less than 30 months even after surgery with curative intent.
\end{abstract}

Keywords: Gastric cancer, Complications, Survival, Mortality

\section{Background}

Although a steady decline in gastric cancer mortality rates over the last few decades is observed, gastric cancer still remains the fourth most common cancer and is the second leading cause of cancer death worldwide with poor survival rates [1]. While incidence rates of gastric cancer in North America, Africa, South and West Asia

\footnotetext{
* Correspondence: a_mickevicius@yahoo.com

${ }^{1}$ Department of Surgery, Lithuanian University of Health Sciences, Eiveniu Str. 2, Kaunas LT-50009, Lithuania

Full list of author information is available at the end of the article
}

are declining, rates in North-East Asia, Eastern part of South America and Eastern Europe stay high [1-3]. Surgery remains the major and potentially curative treatment method for resectable gastric cancer. Considering the location and size of the tumor as well as invasion to the adjacent organs, routinely standard radical total or subtotal gastrectomy with lymphadenectomy or multiorgan resections are performed [4-6]. The overall 5-year survival rate of patients with advanced resectable gastric cancer differs between different countries and different centres, but in general it ranges from $10 \%$ to $30 \%[5,7,8]$. 
Previous studies have shown that age, lymph node and liver metastasis, disease stage and tumour size are important predictive factors for survival in patients with resectable gastric cancer [9-11]. However it is not certainly clear if these predictive factors are the same in all regions and why incidence rates of gastric cancer are still high in the region of Eastern Europe.

The aim of this single centre study was to compare the clinical course and outcomes, such as postoperative complications, the length of hospital stay and mortality rate, over two distinctive time periods.

\section{Methods}

This was a retrospective non-randomized, single center, cohort study. Data collection was performed at the Department of Surgery, Lithuanian University of Health Sciences using specially developed and maintained database from 01-01-1994 to 31-12-2007. During this period 708 patients underwent radical gastrectomy. Five hundred fifty seven consecutive patients were included in the study according to the following inclusion criteria: (1) histologically proven gastric adenocarcinoma; (2) diagnosis based on the UICC TNM staging classification; (3) curative D1 or D2 gastrectomy performed; (4) available complete medical record; (5) postoperative follow-up. Patients with proven distant metastatic disease and in whom only palliative surgery was performed, were excluded from the study. The study population was divided in two groups according to two equal time periods: 01-01-1994 - 31-12-2000 (Group I - 273 patients) and 01-01-2001 - 31-12-2007 (Group II - 284 patients). During the first time period patients diagnosed with gastric cancer were treated according to the guidelines of that time. Standardized protocol was introduced in the year 2001: preoperative evaluation and care (preoperative computed tomography (CT) staging, prophylactic antibiotics), surgical treatment and postoperative care (prophylaxis of thromboembolic disorders; early mobilisation; on day 2 after surgery patients were allowed to drink clear liquids; on postoperative day 3 the soft diet was allowed; drain's placement was at the discretion of the surgeon). The Kaunas Regional Biomedical Research Ethics Committee approved the study (protocol no. BE-2-10) and allowed the use of publicly unavailable database. All patients provided written informed consent. The primary outcome was measured as the five-year survival rate. The gastric cancer- related survival, rates of postoperative complications, the length of hospital stay and 30-day mortality rate were considered as secondary outcomes. The outcomes were studied to evaluate the progress in gastric cancer treatment results over time.

\section{Surgical procedure}

All the surgical procedures were based on the intention to cure. The extent of the surgical procedure was planned based on pre-operative and intra-operative findings, physical condition of the patient. Considering the location of the tumor, routinely standard total (adenocarcinoma involving the proximal third of the stomach) or subtotal (adenocarcinoma of the distal and middle thirds of the stomach) gastrectomy with D1 or D2 lymphadenectomy and a Roux-en-Y reconstruction was performed. Surgical procedures and the definition of

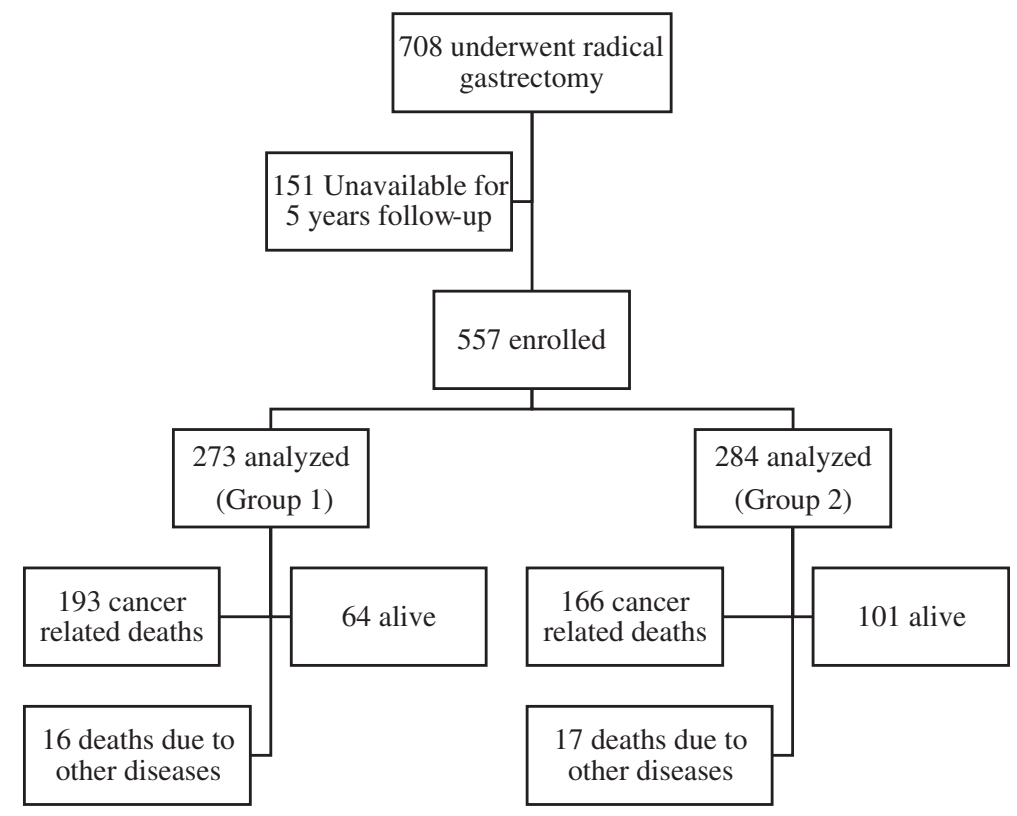

Figure 1 Patient's distribution between groups and subgroups. 
lymphadenectomy referred to the Japanese Classification of Gastric Carcinoma [12]. Combined multiorgan resections were performed in cases of advanced tumors involving the pancreas, colon or spleen. Surgery was considered as a curative when there was no macroscopically residual tumor after surgery and resection margins were histologically clear (R0).

\section{Postoperative course}

Postoperative complications were classified according Clavien-Dindo grading system for the classification of surgical complications (Grade I - V) [13].

Different mainly fluorouracil (5-FU) based adjuvant chemotherapy regiments were inconsistently used postoperatively in the period from 1994 to 2000 . Whereas patients during the period from 2001 to 2007 as a standard received a combined 5-FU and leucovorin adjuvant chemotherapy or concurrent chemoradion treatment (5-FU and leucovorin with 45 Gy radiation dose) in more advanced cancer cases.

\section{Statistical analysis}

Statistical analysis was performed using SPSS 14.0 for Windows (SPSS Inc., Chicago, USA). The data are presented as mean \pm Standard deviation or median and range. The cumulative survival was determined by the Kaplan-Meier method, and univariate comparisons between the groups were performed using the log-rank test. The independent prognostic factors were examined by Cox regression analysis. For comparison between groups, the Mann-Whitney test or Student's t test were employed where appropriate. $\mathrm{P}<0.05$ was considered statistically significant.

\section{Results}

Seven hundred and eight patients with proven gastric adenocarcinoma underwent a subtotal or total gastrectomy

Table 1 Patients' characteristics

\begin{tabular}{lccc}
\hline Parameter & Group I & Group II & P value \\
\hline Gender & $155(56.8 \%)$ & $164(57.7 \%)$ & 0.944 \\
$\quad$ Male & $118(43.2 \%)$ & $120(42.3 \%)$ & 0.938 \\
$\quad$ Female & $63.2 \pm 12.7$ & $64.3 \pm 12.1$ & 0.331 \\
Age & $135(49.5 \%)$ & $134(47.2 \%)$ & 0.767 \\
$\leq 65$ & $138(50.5 \%)$ & $150(52.8 \%)$ & 0.772 \\
$>65$ & & & \\
Procedure & $80(29.3 \%)$ & $52(18.3 \%)$ & 0.019 \\
Total gastrectomy & $193(70.7 \%)$ & $232(81.7 \%)$ & 0.273 \\
Subtotal gastrectomy & $7(2.6 \%)$ & $5(1.8 \%)$ & 0.572 \\
D1 lymphadenectomy & $266(97.4 \%)$ & $279(98.2 \%)$ & 0.952 \\
D2 lymphadenectomy & 273 & 284 & \\
Total & &
\end{tabular}

Table 2 Staging of the disease

\begin{tabular}{lccc}
\hline Parameter & Group I & Group II & P value \\
\hline Pathological stage & & & \\
T1 & $21(7.7 \%)$ & $39(13.7 \%)$ & 0.042 \\
T2 & $42(15.4 \%)$ & $101(35.6 \%)$ & 0.0001 \\
T3 & $118(43.2 \%)$ & $116(40.8 \%)$ & 0.775 \\
T4 & $92(33.7 \%)$ & $28(9.9 \%)$ & 0.0001 \\
N stage & & & \\
N0 & $86(31.5 \%)$ & $91(32.0 \%)$ & 0.931 \\
N1 & $68(24.9 \%)$ & $87(30.6 \%)$ & 0.276 \\
N2 & $92(33.7 \%)$ & $73(25.7 \%)$ & 0.133 \\
N3 & $27(9.9 \%)$ & $33(11.6 \%)$ & 0.589 \\
TNM Stage & & & \\
IA & $18(6.6 \%)$ & $30(10.6 \%)$ & 0.135 \\
IB & $30(11.0 \%)$ & $47(16.5 \%)$ & 0.114 \\
IIA & $43(15.8 \%)$ & $61(21.5 \%)$ & 0.165 \\
IIB & $51(18.7 \%)$ & $47(16.5 \%)$ & 0.586 \\
IIIA & $58(21.2 \%)$ & $65(22.9 \%)$ & 0.765 \\
IIIB & $51(18.7 \%)$ & $24(8.5 \%)$ & 0.002 \\
IIIC & $22(8.1 \%)$ & $10(3.5 \%)$ & 0.044 \\
\hline
\end{tabular}

and D1 or D2 lymphadenectomy with curative intent between 1994 and 2007. One hundred fifty one patients were unavailable for 5 years follow-up. The most frequent detected reason of the unavailability was moving abroad. Data from 557 patients which were followed-up postoperatively was analysed. The distribution of patients between groups and subgroups is shown in Figure 1. There were no significant differences between the groups in gender and age. The number of elderly ( $>65$ years) patients was also similar (50.5\% vs. $52.8 \%$ ). Total gastrectomy was statistically significantly more often performed in Group I (29.3\%) than in Group II (18.3\%). D2 lymphadenectomy was more often performed than D1 lymphadenectomy in both groups (Table 1). Gastric cancer in early stages (IA - IIA)

Table 3 Postoperative course and outcomes

\begin{tabular}{lccc}
\hline Parameter & Group I & Group II & P value \\
\hline Hospital stay (days) & $22.61 \pm 28.96$ & $16.20 \pm 17.01$ & 0.001 \\
Complications* & $55(20.1 \%)$ & $42(14.8 \%)$ & 0.187 \\
I & $13(4.7 \%)$ & $15(5.4 \%)$ & 0.848 \\
II & $8(2.9 \%)$ & $6(2.1 \%)$ & 0.598 \\
III & $26(9.5 \%)$ & $11(3.9 \%)$ & 0.017 \\
IV & $4(1.5 \%)$ & $5(1.7 \%)$ & 1.000 \\
V & $4(1.5 \%)$ & $5(1.7 \%)$ & 1.000 \\
30-day mortality & $15(5.5 \%)$ & $4(1.4 \%)$ & 0.017 \\
\hline *- According to Clavien-Dindo grading system for the classification of surgical \\
complications.
\end{tabular}


Table 4 Postoperative survival analysis

\begin{tabular}{lccc}
\hline Parameter & Group I & Group II & P value \\
\hline Overall survival (months) & $48.40+/-65,966$ & $43.78+/-39.736$ & 0.319 \\
1-year survival rate & $137(50.2 \%)$ & $203(71.5 \%)$ & 0.013 \\
2-years survival rate & $110(40.3 \%)$ & $150(52.8 \%)$ & 0.083 \\
5-year survival rate & $64(23.4 \%)$ & $101(35.6 \%)$ & 0.021 \\
IA & $11(61.1 \%)$ & $25(83.3 \%)$ & 0.644 \\
IB & $22(73.3 \%)$ & $33(70.2 \%)$ & 1.000 \\
IIA & $17(39.5 \%)$ & $23(37.7 \%)$ & 1.000 \\
IIB & $7(13.7 \%)$ & $13(27.7 \%)$ & 0.221 \\
IIIA & $7(12.1 \%)$ & $5(7.7 \%)$ & 0.552 \\
IIIB & 0 & $2(8.3 \%)$ & 0.111 \\
IIIC & 0 & 0 & - \\
Deaths & $209(76.6 \%)$ & $183(64.4 \%)$ & 0.210 \\
Gastric cancer related & $193(92.3 \%)$ & $166(90.7 \%)$ & 0.942 \\
Other causes & $16(7.7 \%)$ & $17(9.3 \%)$ & 0.717 \\
\hline
\end{tabular}

was more frequently diagnosed in Group II than in Group I and in late stages (IIB - IIIC) more frequently in Group I. Statistically significant difference was found only when comparing stages IIIB-IIIC. Significantly more patients were diagnosed with lower stage of the primary tumor
(T stage) in Group II ( $13.7 \%$ vs $7.7 \%$ in $\mathrm{T} 1$ stage $(\mathrm{P}=0.04)$ and $35.6 \%$ vs $15.4 \%$ in $\mathrm{T} 2$ stage $(\mathrm{P}=0.0001))$. On the contrary in Group I more patients were diagnosed in T4 stage (33.7\% vs $9.9 \%, \mathrm{P}=0.0001$ ) (Table 2 ).

When analyzing postoperative course of the disease shorter hospital stay $(16.20 \pm 17.01$ vs. $22.61 \pm 28.96$ days, $\mathrm{P}=0.001)$ and lower 30 -day mortality rate $(1.4 \%$ vs $5.5 \%$, $\mathrm{P}=0.0173)$ was identified in Group II. During postoperative period in 6 patients (2.2\%) of Group I and in 7 patients (2.5\%) of Group II anastomotic leakage was identified. However grade of postoperative complications was similar between both groups. Only Grade III (Complications requiring surgical, endoscopic or radiological intervention) complications were statistically significant more often identified in Group I (9.5\% vs 3.9\%, P = 0.017) (Table 3).

The survival analysis revealed higher median overall survival (months) in Group I (48.40 \pm 65.966 vs. $43.78 \pm$ 39.736). However Group I patients are observed for a longer time period and long-term survivors among them could influence this outcome. In contrary when analysing 1-year and 5-year survival rates, significantly higher survival is observed in Group II $(71.5 \%$ vs. $50.2 \%$ and $35.6 \%$ vs. $23.4 \%$ ). Patients with more advanced T stage and involved lymphnodes had worse 5-year survival prognosis as compared with patients with les advanced

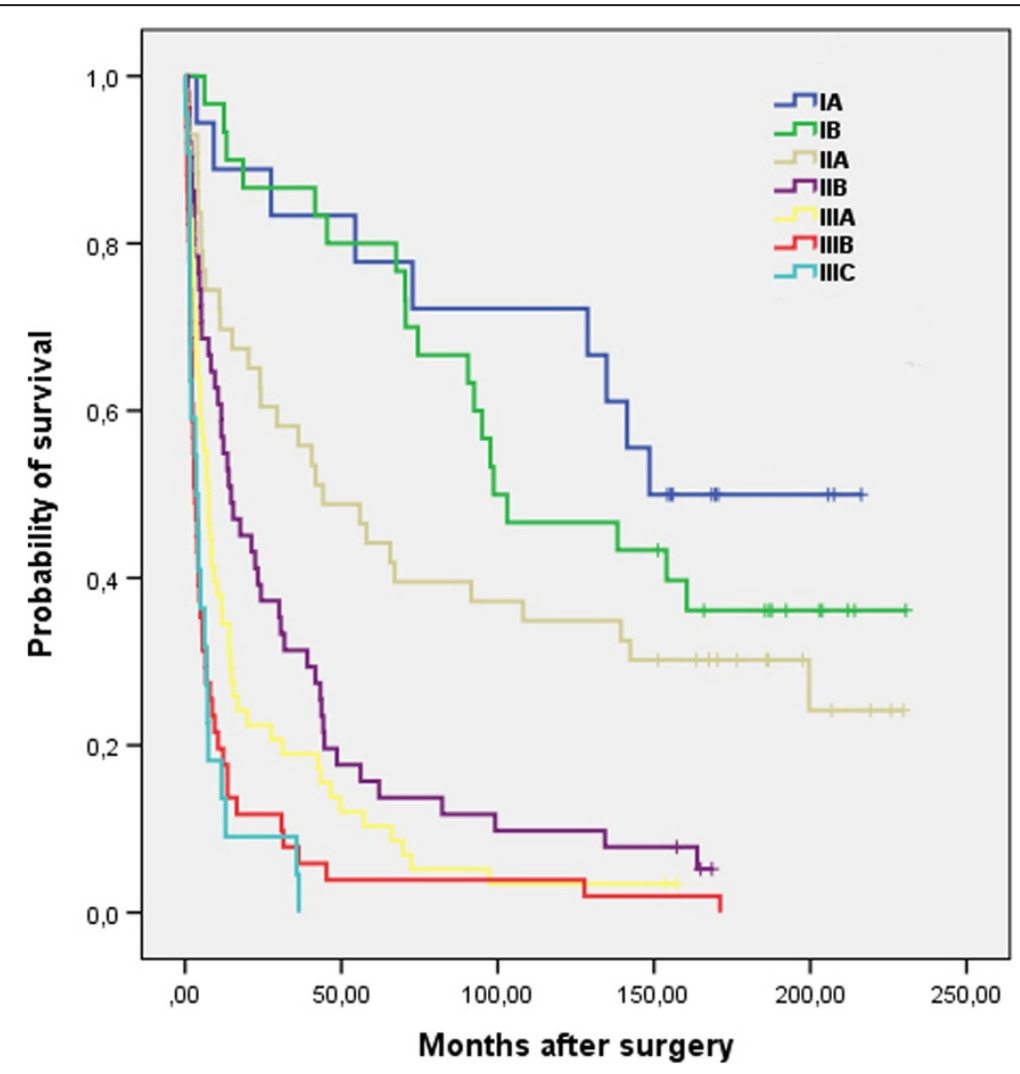

Figure 2 Overall survival (in months) after surgery according to TNM stage (Group I). 
$\mathrm{T}$ stage and no lymphnodes involvement. However statistically significant difference was found only when analyzing lymphnodes involvement $(\mathrm{p}<0.05)$.

In both groups the reasons of death were similar; the majority of patients died of gastric cancer (92.3\% vs. $90.7 \%)$. There was no difference in 5-year survival rate when comparing different TNM stages between both groups (Table 4). However in early stages (IA - IIA) survival rate was higher comparing with advanced stages (IIB - IIIC) (Figures 2 and 3).

\section{Discussions}

The incidence of gastric cancer in Eastern Europe remains high following Eastern Asia and South America. The highest gastric cancer incidence rate in European Union (EU) is reported in Lithuania [14]. In this singleinstitutional study we present case series from large university hospital in Lithuania raising a question: is there a significant progress in terms of treatment success and survival among patients diagnosed with gastric cancer and treated with curative surgery over time? To clarify changes, positive or negative tendencies the two groups on background of time of surgery were created.

Most of demographic data, clinicopathologic characteristics in our study are comparable to the groups presented from other European countries. Regrettably, gastric cancer remains often diagnosed in advanced stages in Lithuania, leading to poor prognosis. Late diagnosis of gastric cancer is a well-known problem among patients from Western countries. Hundahl et al. [7] from United States (US) report $65 \%$ of gastric cancers presenting at an advanced stage (T3-T4) with a nearly $85 \%$ of tumours accompanied with lymph node affection at the time of surgery. The data are very close to ours (T3-T4-63.6\%; N+68.2\%). However higher gastric cancer incidence rate in Lithuania leads to even more actual problem.

The interesting difference identified between our data and studies done in Western Europe - a lack of growing incidence of upper-third gastric cancer. In contrary, we even had more distal and middle third tumours and higher proportion of patients underwent subtotal gastrectomy in Group II. Although we have not analysed this factor in our study, high prevalence of Helicobacter pylori infection in Lithuania (78.5\% in the year 1999 and $69.7 \%$ in the year 2005) could be related to the high incidence of gastric cancer [15].

The overall incidence of directly surgery-related postoperative complications in our study (anastomosis or duodenal stump leakage) $<3 \%$ is comparable to majority of published data [16-18]. The rate and grade of postoperative

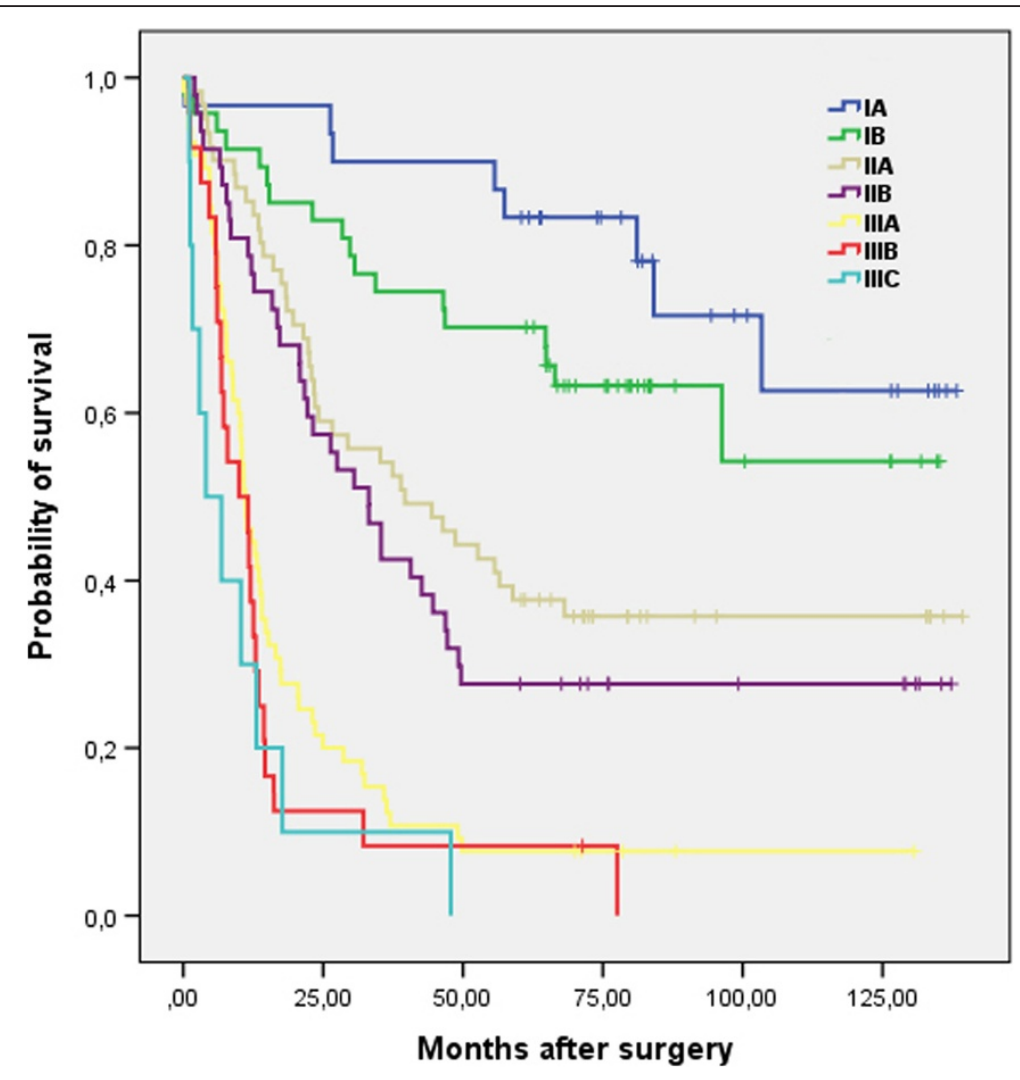

Figure 3 Overall survival (in months) after surgery according to TNM stage (Group II). 
complications (except Grade III complications) in our study was similar in both groups; however 30-day mortality rate and in-hospital stay decreased significantly in Group II. These results should be considered as a consequence of more detailed preoperative patients' selection, standardised surgery technique, and improved perioperative care over time.

The overall 5-year survival rate was slightly higher in Group II, however remaining below 40\% in entire cohort. The higher survival rate possibly caused by a higher rate of early detection (standardized protocol of diagnosis) of gastric cancer (more T1, T2 tumours, less IIIB, IIIC stages in Group II), perioperative care improvement over time and possibilities of palliation procedures in cases of recurrent disease. The most common cause of treatment failure in our study was peritoneal recurrence and spread of the disease. Similar data are presented by other authors $[19,20]$. Observed 5-year survival rate in early stages (IA $61.1 \%$ vs. $83.3 \%$ and IB $73.3 \%$ vs. $70.2 \%$ ) of gastric cancer is lower than in Eastern countries, but is similar to the data presented by the Western European countries from the similar time period [21,22].

Regarding the surgical technique and extent of lymph node dissection, it became highly standardised over last 15 years. The patients in our study mainly underwent gastrectomy with a Roux-en-Y reconstruction and D2 lymphadenectomy ( $97.4 \%$ vs. $98.2 \%$ ). There has been controversy regarding the extent of lymph node dissection around the European centres in last decade, pointing on higher postoperative morbidity after D2 dissection, however most experts suggest that extended lymphadenectomy could be performed with acceptable morbidity and mortality rate by specialized surgeons in large-volume centres [23-25].

\section{Conclusions}

Despite some positive changes in early postoperative mortality rate, hospital stay and overall survival over the time, gastric cancer treatment results remain poor. Prognosis of treatment of gastric cancer depends mainly on the stage of the disease. Absence of screening programs and lack of clinical symptoms in early stages of gastric cancer lead to circumstances when most of the patients presenting with advanced stage of the disease can expect a median survival of less than 30 months even after curative intent surgery. The most efficient way to reach more significant progress in gastric cancer treatment should concentrate mostly on earlier diagnosis, when survival results after radical surgery are far more promising.

\section{Competing interests}

The authors declare that they have no competing interests.

\section{Authors' contributions}

AM, PI, RM, AP, DB, MK, ZE collected, analysed and interpreted the data. AM, AM, ZD drafted the study concept and design, supervised the study, analysed and interpreted the data. AM, PI, ZD drafted the manuscript. All authors read and approved the final manuscript.

\section{Author details}

${ }^{1}$ Department of Surgery, Lithuanian University of Health Sciences, Eiveniu Str. 2, Kaunas LT-50009, Lithuania. 'Laboratory of Surgical Gastroenterology, Institute for Digestive System Research, Lithuanian University of Health Sciences, Eiveniu str. 2, Kaunas LT-50009, Lithuania. ${ }^{3}$ Department of Oncology, Lithuanian University of Health Sciences, Eiveniu str. 2, Kaunas LT-50009, Lithuania.

Received: 28 May 2014 Accepted: 17 November 2014

Published: 26 November 2014

\section{References}

1. Parkin DM, Bray F, Ferlay J, Pisani P: Global cancer statistics, 2002. CA Cancer J Clin 2005, 55(2):74-108

2. Yamaoka Y, Kato M, Asaka M: Geographic differences in gastric cancer incidence can be explained by differences between Helicobacter pylori strains. Intern Med 2008, 47(12):1077-1083.

3. Smailyte $\mathrm{G}$, Kurtinaitis J: Cancer mortality differences among urban and rural residents in Lithuania. BMC Public Health 2008, 8:56.

4. Fukuda N, Sugiyama Y, Wada J: Prognostic factors of T4 gastric cancer patients undergoing potentially curative resection. WJG 2011, 17(9):1180-1184.

5. Dikken JL, van de Velde CJ, Coit DG, Shah MA, Verheij M, Cats A: Treatment of resectable gastric cancer. Ther Adv Gastroenterol 2012, 5(1):49-69.

6. Lee $\mathrm{JH}$, Kim KM, Cheong $\mathrm{JH}$, Noh SH: Current management and future strategies of gastric cancer. Yonsei Med J 2012, 53(2):248-257.

7. Hundahl SA, Phillips JL, Menck HR: The National Cancer Data Base Report on poor survival of U.S. gastric carcinoma patients treated with gastrectomy: Fifth Edition American Joint Committee on Cancer staging, proximal disease, and the "different disease" hypothesis. Cancer 2000, 88(4):921-932.

8. Cenitagoya GF, Bergh CK, Klinger-Roitman J: A prospective study of gastric cancer. 'Real' 5 -year survival rates and mortality rates in a country with high incidence. Digestive surgery 1998, 15(4):317-322.

9. Shiraishi N, Sato K, Yasuda K, Inomata M, Kitano S: Multivariate prognostic study on large gastric cancer. J Surg Oncol 2007, 96(1):14-18.

10. Seo SH, Hur H, An CW, Yi X, Kim JY, Han SU, Cho YK: Operative risk factors in gastric cancer surgery for elderly patients. J Gastric Cancer 2011, 11(2):116-121.

11. Smith JK, McPhee JT, Hill JS, Whalen GF, Sullivan ME, Litwin DE, Anderson FA, Tseng JF: National outcomes after gastric resection for neoplasm. Arch Surg 2007, 142(4):387-393.

12. Japanese Gastric Cancer A: Japanese classification of gastric carcinoma: 3rd English edition. Gastric Cancer 2011, 14(2):101-112.

13. Clavien PA, Barkun J, de Oliveira ML, Vauthey JN, Dindo D, Schulick RD, de Santibanes E, Pekolj J, Slankamenac K, Bassi C, Graf R, Vonlanthen R, Padbury $\mathrm{R}$, Cameron JL, Makuuchi M: The Clavien-Dindo classification of surgical complications: five-year experience. Ann Surg 2009, 250(2):187-196.

14. Levi F, Lucchini F, Negri E, La Vecchia C: Trends in mortality from major cancers in the European Union, including acceding countries, in 2004. Cancer 2004, 101(12):2843-2850.

15. Jonaitis L, Ivanauskas A, Janciauskas D, Funka K, Sudraba A, Tolmanis I, Krams A, Stirna D, Vanags A, Kupcinskas L, Leja M, Lin JT: Precancerous gastric conditions in high Helicobacter pylori prevalence areas: comparison between Eastern European (Lithuanian, Latvian) and Asian (Taiwanese) patients. Medicina 2007, 43(8):623-629.

16. Gockel I, Pietzka S, Gonner U, Hommel G, Junginger T: Subtotal or total gastrectomy for gastric cancer: impact of the surgical procedure on morbidity and prognosis-analysis of a 10-year experience. Langenbecks Arch Surg 2005, 390(2):148-155.

17. Oh SJ, Choi WB, Song J, Hyung WJ, Choi SH, Noh SH, Yonsei Gastric Cancer $\mathrm{C}$ : Complications requiring reoperation after gastrectomy for gastric cancer: 17 years experience in a single institute. J Gastrointest Surg 2009, 13(2):239-245.

18. Otsuji E, Fujiyama J, Takagi T, Ito T, Kuriu Y, Toma A, Okamoto K, Hagiwara A, Yamagishi $H$ : Results of total gastrectomy with extended lymphadenectomy for gastric cancer in elderly patients. J Surg Oncol 2005, 91(4):232-236. 
19. Yoo CH, Noh SH, Shin DW, Choi SH, Min JS: Recurrence following curative resection for gastric carcinoma. Br J Surg 2000, 87(2):236-242.

20. Moriguchi S, Maehara Y, Korenaga D, Sugimachi K, Nose Y: Risk factors which predict pattern of recurrence after curative surgery for patients with advanced gastric cancer. Surg Oncol 1992, 1(5):341-346.

21. Lamb P, Sivashanmugam T, White M, Irving M, Wayman J, Raimes S: Gastric cancer surgery-a balance of risk and radicality. Ann R Coll Surg Engl 2008, 90(3):235-242.

22. Bonenkamp JJ, Hermans J, Sasako M, van de Velde CJ, Welvaart K, Songun I, Meyer S, Plukker JT, Van Elk P, Obertop H, Gouma DJ, Van Lanschot JJ,

Taat CW, De Graaf PW, Von Meyenfeldt MF, Tilanus H, Dutch Gastric Cancer Group: Extended lymph-node dissection for gastric cancer. N Engl J Med 1999, 340(12):908-914

23. Hartgrink $\mathrm{HH}$, van de Velde $\mathrm{CJ}$, Putter $\mathrm{H}$, Bonenkamp JJ, Klein Kranenbarg E, Songun I, Welvaart K, van Krieken JH, Meijer S, Plukker JT, Van Elk PJ,

Obertop H, Gouma DJ, Van Lanschot JJ, Taat CW, De Graaf PW, Von Meyenfeldt MF, Tilanus H, Sasako M: Extended lymph node dissection for gastric cancer: who may benefit? Final results of the randomized Dutch gastric cancer group trial. J Clin Oncol 2004, 22(11):2069-2077.

24. Cuschieri A, Weeden S, Fielding J, Bancewicz J, Craven J, Joypaul V, Sydes M, Fayers P: Patient survival after D1 and D2 resections for gastric cancer: long-term results of the MRC randomized surgical trial. Surg Co-operative Group. Br J Cancer 1999, 79(9-10):1522-1530.

25. Songun I, Putter H, Kranenbarg EM, Sasako M, van de Velde CJ: Surgical treatment of gastric cancer: 15-year follow-up results of the randomised nationwide Dutch D1D2 trial. Lancet Oncol 2010, 11(5):439-449.

doi:10.1186/1471-2482-14-98

Cite this article as: Mickevicius et al:: Trends and results in treatment of gastric cancer over last two decades at single East European centre: a cohort study. BMC Surgery 2014 14:98.

\section{Submit your next manuscript to BioMed Central and take full advantage of:}

- Convenient online submission

- Thorough peer review

- No space constraints or color figure charges

- Immediate publication on acceptance

- Inclusion in PubMed, CAS, Scopus and Google Scholar

- Research which is freely available for redistribution 